\title{
Contributions of Tzero ${ }^{\mathrm{TM}}$ technology to temperature modulated Differential scanning calorimetry
}

\author{
J. Loubens, and F. Hoppenot \\ TA Instruments, Thermal Analysis Applications Laboratory, Guyancourt, France
}

Differential Scanning Calorimetry (DSC) is the most used of thermal analysis techniques. Temperature Modulated DSC is a technique which has extended a lot the applications of DSC because of new possibilities like improved separation of overlapped transitions, a higher sensitivity and an improved accuracy of heat capacity measurements.

Basic principle of Modulated DSC is to apply two heating rates simultaneously and consequently to measure the heat flow obtained by this solicitation. The average underlying heating rate gives the same heat flow than conventional DSC (called total heat flow), while the sinusoidal instantaneous heating rate allows the separation of this total heat flow into two components, one heat flow linearly dependent of the heating rate change, and one heat flow dependent on time and temperature, and not dependent of the heating rate change. This technique allows a direct measurement of heat capacity, and the separation of thermal events due to a heat capacity change (for instance glass transition) from kinetic events (polymerization, dehydration) because of the purely sinusoidal thermal solicitation.

Tzero $^{\mathrm{TM}}$ technology has been specifically developed to quantify the instrument contribution to the DSC measurement; and offers a much more accurate representation of the absorbed or evolved heat flow by the analyzed material. This technique directly measures the heat transfers through the thermal sensors (thermal resistances and thermal capacitances of the sample and reference platforms) and consequently two independent measurements of sample and reference heat flows. The result is the improved resolution of thermal peaks and a quick return to baseline after a transition.

Advanced Tzero ${ }^{\mathrm{TM}}$ technology takes in account heat transfers through sample and reference pans in the heat flow measurement. This allows Modulated DSC experiments at average heating rates similar to conventional DSC, typically $10 \mathrm{~K} / \mathrm{min}$.

\section{References}

1. G.W.H. Hohne, W. Hemminger, H.-J. Flammersheim, Differential Scanning Calorimetry- An Introduction for Practitioners, Ed: Springer (2004)

2. R.L. Danley, Thermochimica Acta 395201 (2003)

3. E. Dantras, J. Dandurand, C. Lacabanne, Macromolecules 35-6 2090 (2001)

4. L. Carpentier, O. Bustin, M. Descamps, J.Phys.D: Appl. Phys. 35402 (2002) 